\title{
(6) OPEN ACCESS \\ Changes in intraocular pressure in study and fellow eyes in the IVAN trial
}

\author{
Alexander J E Foss, ${ }^{1}$ Lauren J Scott, ${ }^{2}$ Chris A Rogers, ${ }^{2}$ Barney C Reeves, ${ }^{2}$ \\ Faruque Ghanchi, ${ }^{3}$ Jonathan Gibson, ${ }^{4}$ Usha Chakravarthy, ${ }^{5}$ On behalf of the IVAN \\ study group
}

- Additional material is published online only. To view please visit the journal online (http://dx.doi.org/10.1136/ bjophthalmol-2015-307595).

${ }^{1}$ Department of Ophthalmology, Queen's Medical Centre, Nottingham, UK

${ }^{2}$ School of Clinical Sciences, Clinical Trials Evaluation Unit, University of Bristol, Bristol, UK

${ }^{3}$ Department of

Ophthalmology, Bradford Royal Infirmary, Bradford, West Yorkshire, UK

${ }^{4}$ School of Life and Health Sciences, Aston University, Birmingham, UK

${ }^{5}$ Centre for Experimental Medicine, Queens University Belfast, Belfast, UK

\section{Correspondence to} Dr Chris A Rogers, Clinical Trials Evaluation Unit, University of Bristol, Bristol, UK; chris.rogers@bristol.ac.uk

Received 16 August 2015 Revised 31 January 2016 Accepted 22 February 2016 Published Online First 12 April 2016

\section{ABSTRACT}

Purpose To describe changes in intraocular pressure (IOP) in the 'alternative treatments to /nhibit VEGF in Age-related choroidal Neovascularisation (IVAN)' trial (registered as ISRCTN92166560).

Design Randomised controlled clinical trial with factorial design.

Participants Patients $(n=610)$ with treatment naive neovascular age-related macular degeneration were enrolled and randomly assigned to receive either ranibizumab or bevacizumab and to two regimens, namely monthly (continuous) or as needed (discontinuous) treatment.

Methods At monthly visits, IOP was measured preinjection in both eyes, and postinjection in the study eye.

Outcome measures The effects of 10 prespecified covariates on preinjection IOP, change in IOP (postinjection minus preinjection) and the difference in preinjection IOP between the two eyes were examined.

Results For every month in trial, there was a statistically significant rise in both the preinjection IOP and the change in IOP postinjection during the time in the trial (estimate $0.02 \mathrm{~mm} \mathrm{Hg}, 95 \% \mathrm{Cl} 0.01$ to 0.03 , $\mathrm{p}<0.001$ and $0.03 \mathrm{~mm} \mathrm{Hg}, 95 \% \mathrm{Cl} 0.01$ to 0.04 , $p=0.002$, respectively). There was also a small but significant increase during the time in trial in the difference in IOP between the two eyes (estimate $0.01 \mathrm{~mm} \mathrm{Hg}, 95 \% \mathrm{Cl} 0.005$ to $0.02, \mathrm{p}<0.001)$. There were no differences between bevacizumab and ranibizumab for any of the three outcomes ( $p=0.93$, $p=0.22$ and $p=0.87$, respectively).

Conclusions Anti-vascular endothelial growth factor agents induce increases in IOP of small and uncertain clinical significance.

Trial registration number ISRCTN92166560.

\section{INTRODUCTION}

A rise in intraocular pressure (IOP) was not observed in the initial ANCHOR and MARINA trials of agents that inhibit vascular endothelial growth factor (VEGF); but subsequently small clinical series have reported rises in IOP in around $3 \%{ }^{12}$ to $12 \%{ }^{3}$ in treated eyes with similar rates observed in the fellow eye. ${ }^{4}$ Concerns have been raised that there is a cumulative effect on pressure rise after multiple intraocular injections of anti-VEGF agents, ${ }^{5-7}$ although this finding has not been consistently replicated. ${ }^{8}$ Mechanisms that have been postulated to explain this IOP rise include the volume effect of the injection, ${ }^{9}$ the particular properties of the agent (bevacizumab is a full length antibody with an Fc fragment which may induce a trabeculitis, ${ }^{10-12}$ and/ or the presence of particulates) or the inhibition of VEGF within the trabecular meshwork leading to reduced facility of aqueous outflow

Accordingly, we undertook an exploratory analysis of the alternative treatments in the inhibition of VEGF in age-related choroidal neovascularisation (IVAN) clinical trial dataset. The IVAN trial (registered ISRCTN92166560) was a randomised controlled trial with a $2 \times 2$ factorial design comparing ranibizumab with bevacizumab and monthly (continuous) with as needed (discontinuous) treatment strategies.

We hypothesised that:

- Preinjection IOP would increase with the number of injections administered.

- The size of the postinjection pressure 'spike' would provide an estimate of the functioning of the trabecular meshwork and facility of aqueous outflow.

As IOP was measured in both eyes at every visit, the difference in IOP between study and fellow eyes over time could also be examined, allowing the fellow eye to act as a within-subject control for a trend in preinjection IOP.

\section{MATERIALS AND METHODS \\ Patients}

Patients over the age of 50 with active, treatment naive neovascular age-related macular degeneration (nAMD) were recruited. Those with field defects attributable to glaucoma were excluded (guidance rather than formal exclusion-there being no reliable way to distinguish field defects due to glaucoma from those due to macular degeneration) but those with a history of ocular hypertension, or glaucoma per se, were not (ie, these patients are best considered as having preperimetric glaucoma). The CONSORT diagram is reported elsewhere. ${ }^{13}$ The full list of inclusion and exclusion criteria is provided as online supplementary file 1. A UK National Health Service Research Ethics Committee approved the trial (07/NIR03/37). Patients with glaucoma were identified on the basis of either having had glaucoma surgery (of which there were none) or prescription of topical pressure lowering medications (46 patients). This criterion does not distinguish between patients with ocular hypertension and those with glaucoma and so these two groups were combined into a single group and labelled as glaucoma for the analyses. And it should be noted that neither gonioscopy nor central corneal thickness measurements were performed. 


\section{IOP measurements}

The IOP was measured monthly using Goldmann applanation tonometry in both eyes prior to treatment and in the study eye after injection if treated. Two readings were made and if more than $2 \mathrm{~mm}$ apart, a third reading was taken. Visit IOP for each eye was the mean of the two or the median of the three. Equipment was calibrated at least twice annually.

The clinicians were not masked to which was the study eye for these measurements but were doing it for safety monitoring only and no hypothesis was even discussed until after data collection was complete.

\section{Study outcomes}

Three outcomes were prespecified in an analysis plan. These were as follows:

1. Postinjection IOP 'spike' (ie, postinjection IOP minus preinjection IOP) in the study eye, at visits where injections were administered.

2. Preinjection IOP in the study eye.

3. The difference in preinjection IOP between the study and fellow eyes (ie, study eye preinjection IOP minus fellow eye preinjection IOP).

\section{Statistical analysis}

The analyses used data from all available IVAN study visits (up to 25 visits). Ten covariates were prespecified in the analysis plan: months in study, months since last injection, mean arterial blood pressure, study drug, cataract surgery, glaucoma, age at randomisation, gender, baseline preinjection IOP in the study eye (outcomes 1 and 3) and the time between the injection and the postinjection IOP measurement (outcome 1). We treated time in trial as a proxy for number of injections (adjusting for time since last injection) since the two were strongly correlated. Linear mixed modelling was used to analyse the three study outcomes; intercept and 'months in trial' terms were fitted as random effects in all three models. For the analyses of preinjection IOP, namely outcomes 2 and 3, the baseline measurements of each outcome were modelled jointly with the subsequent values to avoid the need to exclude cases with missing baseline values. Model validity was checked and outlying values were excluded. Linearity assumptions were also checked and when non-linear, the data were grouped into categories. All covariates were retained in the models regardless of their significance. Results are reported as effect estimates with $95 \%$ CIs. Two-sided $\mathrm{p}$ values $<0.05$ are considered statistically significant.

IOP was measured at every visit and the amount of missing data was low (less than 5\% of attended visits; a further $13 \%$ of visits were not attended ${ }^{13}$ ). However, blood pressure was only measured every 3 months. Plots of the mean arterial pressure suggested that the pressure did not change much over time, so values were interpolated for the visits where it was not measured.

Cataract surgery was identified by the phakic status of the eye. A time-dependent indicator variable was fitted to denote when surgery took place. The impact of having surgery in both eyes was tested by adding an interaction term to the model. This term was retained if significant at the 5\% level. As for cataract surgery, a time-dependent indicator variable was fitted to describe when patients' were identified as glaucomatous during time in the trial.

Baseline preinjection IOP in the study eye, study drug, age at randomisation and gender did not vary across visits.

\section{RESULTS}

All 610 participants in the IVAN study cohort were included in this analysis. ${ }^{13}$ There were 13371 study visits including baseline and injections were administered at 10160 of these visits.

The median duration of active participation in the trial was 23.6 months (IQR 22.8 to 24.3), with a median time between injections of 1.0 month (IQR 0.9 to 1.2 ). Baseline preinjection IOP in the study eye was measured for $607 / 610$ patients with a median of $15 \mathrm{~mm} \mathrm{Hg}$ (IQR 14 to 18 ). Glaucoma was present in $46 / 610$ (7.5\%) patients, and 219/610 (35.9\%) patients were pseudophakic/aphakic in at least one eye during the trial; the majority of patients classified as glaucomatous (37/46 (80.4\%) or pseudophakic/aphakic (181/219 (82.6\%) were classified as such at trial entry. Participant demographics and ophthalmic characteristics are summarised in table 1.

Participant age at randomisation was grouped into categories as the relationship with outcomes was non-linear.

\section{Outcome 1: spikes in postinjection IOP (postinjection IOP minus preinjection IOP)}

IOP was measured preinjection and postinjection for 10009 of the 10160 (98.5\%) injections and the median postinjection IOP spike was $+3 \mathrm{~mm} \mathrm{Hg}$ (IQR 0 to 7 ).

Figure 1 shows the multivariable effect estimates (ie, adjusted for other covariates in the model) for the covariates in the model examining the factors associated with postinjection IOP spike.

For every month in the trial, on average a patient's postinjection IOP increased by $0.03 \mathrm{~mm} \mathrm{Hg}$ (95\% CI 0.01 to 0.04 , $\mathrm{p}=0.002$ ). In addition, the longer the time interval between injection administration and measurement of postinjection IOP, the lower the IOP spike $(-0.07 \mathrm{~mm} \mathrm{Hg} / \mathrm{min})$.

Compared with no cataract surgery, the IOP spike was reduced in cataract-operated study eyes $(-1.00 \mathrm{~mm} \mathrm{Hg}, 95 \% \mathrm{CI}-1.75$ to $-0.25)$, but did not differ when fellow eyes only or both eyes had experienced surgery (figure 1 ; test for eye interaction $\mathrm{p}=0.028$ ).

Glaucoma in either eye increased the postinjection IOP spike on average by $1.15 \mathrm{~mm} \mathrm{Hg}$. The relationship with age was nonlinear, but those aged 85 and above had the highest IOP spike compared with the reference group of age 50-69 years.

Male patients had a larger postinjection IOP spike than females.

Months since last injection, mean arterial pressure and study drug had no statistically significant effect on postinjection IOP change and the use of bevacizumab resulted in a non-significant but lower IOP spike than ranibizumab.

\section{Outcome 2: preinjection IOP in the study eye during time in study}

IOP in the study eye was measured prior to injection at 13281 (99.3\%) of the 13371 attended study visits. Data were available for 607 patients at visit 0 and 514 patients at visit 24. The median preinjection IOP across all visits was $15 \mathrm{~mm} \mathrm{Hg}$ (IQR 13 to 18 ). Figure 2 shows the multivariable effect estimates for covariates associated with preinjection IOP.

The average preinjection IOP in the study eye increased by $0.02 \mathrm{~mm} \mathrm{Hg} / \mathrm{month}$. For every unit increase of mean arterial pressure, preinjection IOP increased by $0.01 \mathrm{~mm} \mathrm{Hg}$. Compared with no cataract surgery, study eye surgery significantly reduced preinjection IOP $(-1.42 \mathrm{~mm} \mathrm{Hg})$, as did surgery on both eyes $(-0.79 \mathrm{~mm} \mathrm{Hg})$, but surgery on the fellow eye alone had no effect $(-0.18 \mathrm{~mm} \mathrm{Hg}$; test for eye interaction $\mathrm{p}=0.028)$.

Months since last injection, study drug, glaucoma status and gender had no statistically significant effect on preinjection IOP $(\mathrm{p}=0.76, \mathrm{p}=0.93, \mathrm{p}=0.47$ and $\mathrm{p}=0.32$, respectively). 
Table 1 Summary of description of study population by glaucoma status

\begin{tabular}{|c|c|c|c|c|}
\hline Patient characteristics & $\mathbf{n}$ & Glaucoma $(n=46)$ & No glaucoma $(n=564)$ & Overall $(n=610)$ \\
\hline \multicolumn{5}{|l|}{ Baseline } \\
\hline Drug (Avastin) & 610 & $23(50.0 \%)$ & $273(48.4 \%)$ & $296(48.5 \%)$ \\
\hline Age (years) & 610 & $80.0(7.1)$ & $77.5(7.4)$ & $77.7(7.4)$ \\
\hline $50-69$ & & $2(4.3 \%)$ & $90(16.0 \%)$ & $92(15.1 \%)$ \\
\hline $70-74$ & & $13(28.3 \%)$ & $97(17.2 \%)$ & $110(18.0 \%)$ \\
\hline $75-79$ & & $8(17.4 \%)$ & $151(26.8 \%)$ & $159(26.1 \%)$ \\
\hline $80-84$ & & $7(15.2 \%)$ & $132(23.4 \%)$ & $139(22.8 \%)$ \\
\hline $85+$ & & $16(34.8 \%)$ & $94(16.7 \%)$ & $110(18.0 \%)$ \\
\hline Gender (male) & 610 & $18(39.1 \%)$ & $226(40.1 \%)$ & $244(40.0 \%)$ \\
\hline Preinjection IOP in study eye & 607 & $16.8(3.8)$ & $15.3(3.1)$ & $15.4(3.2)$ \\
\hline Postinjection IOP change in study eye & 594 & $2.8(6.5)$ & $3.1(5.6)$ & $3.1(5.7)$ \\
\hline Preinjection IOP eye difference (study eye IOP minus fellow eye IOP) & 606 & $-0.7(3.8)$ & $0.0(1.6)$ & $-0.1(1.9)$ \\
\hline \multicolumn{5}{|l|}{ During the trial } \\
\hline Any cataract surgery (pseudo/aphakic) & 610 & $27(58.7 \%)$ & $192(34.0 \%)$ & $219(35.9 \%)$ \\
\hline In study eye only & & $7(25.9 \%)$ & $39(20.3 \%)$ & $46(21.0 \%)$ \\
\hline In fellow eye only & & $3(11.1 \%)$ & $29(15.1 \%)$ & $32(14.6 \%)$ \\
\hline In both eyes & & $17(63.0 \%)$ & $124(64.6 \%)$ & $141(64.4 \%)$ \\
\hline Months in trial & 610 & 23.5 (23.0 to 24.5$)$ & 23.6 (22.8 to 24.3$)$ & 23.6 (22.8 to 24.3$)$ \\
\hline Months between injections & 13371 & $1.0(0.9$ to 1.8$)$ & $1.0(0.9$ to 1.2$)$ & $1.0(0.9$ to 1.2$)$ \\
\hline Time (min) between injection and postinjection IOP measurement & 9966 & 25 (15 to 33) & 21 (12 to 32$)$ & 22 (13 to 32$)$ \\
\hline Mean arterial pressure $(\mathrm{mm} \mathrm{Hg})$ & 4795 & $95.4(10.6)$ & $96.3(10.9)$ & $96.2(10.9)$ \\
\hline Preinjection IOP in study eye & 13281 & $16.6(4.0)$ & $15.3(3.3)$ & $15.4(3.4)$ \\
\hline Postinjection IOP change in study eye & 10009 & $4.4(6.9)$ & $3.8(5.3)$ & $3.8(5.4)$ \\
\hline Preinjection IOP eye difference (study eye IOP minus fellow eye IOP) & 13,208 & $-0.4(5.0)$ & $0.1(1.8)$ & $0.5(2.3)$ \\
\hline \multicolumn{5}{|l|}{ At visit 24} \\
\hline Preinjection IOP in study eye & 514 & $17.0(4.0)$ & $15.7(3.3)$ & $15.8(3.4)$ \\
\hline Postinjection IOP change in study eye (at visit 23) & 358 & $5.1(4.1)$ & $3.9(5.1)$ & $4.0(5.0)$ \\
\hline Preinjection IOP eye difference (study eye IOP minus fellow eye IOP) & 510 & $0.5(2.2)$ & $0.2(1.9)$ & $0.2(1.9)$ \\
\hline
\end{tabular}

IOP, intraocular pressure.

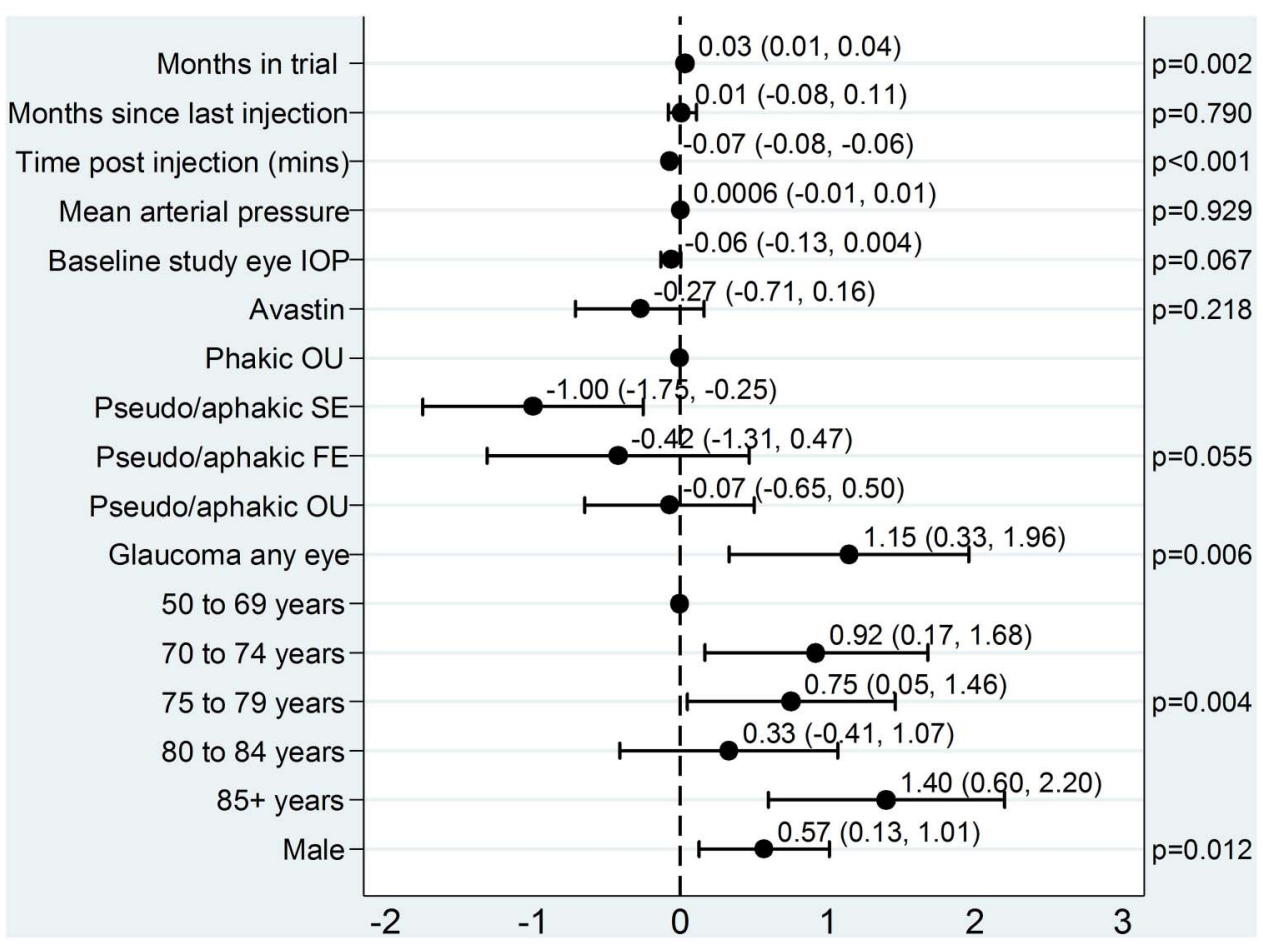

Figure 1 Model for spikes in postinjection IOP (postinjection minus preinjection) in the study eye. Plot of the effects of covariates on IOP spikes in injected study eyes. The point estimates and $95 \% \mathrm{Cls}$ are shown. FE, fellow eye; IOP, intraocular pressure; OU, both eyes; SE, study eye. 


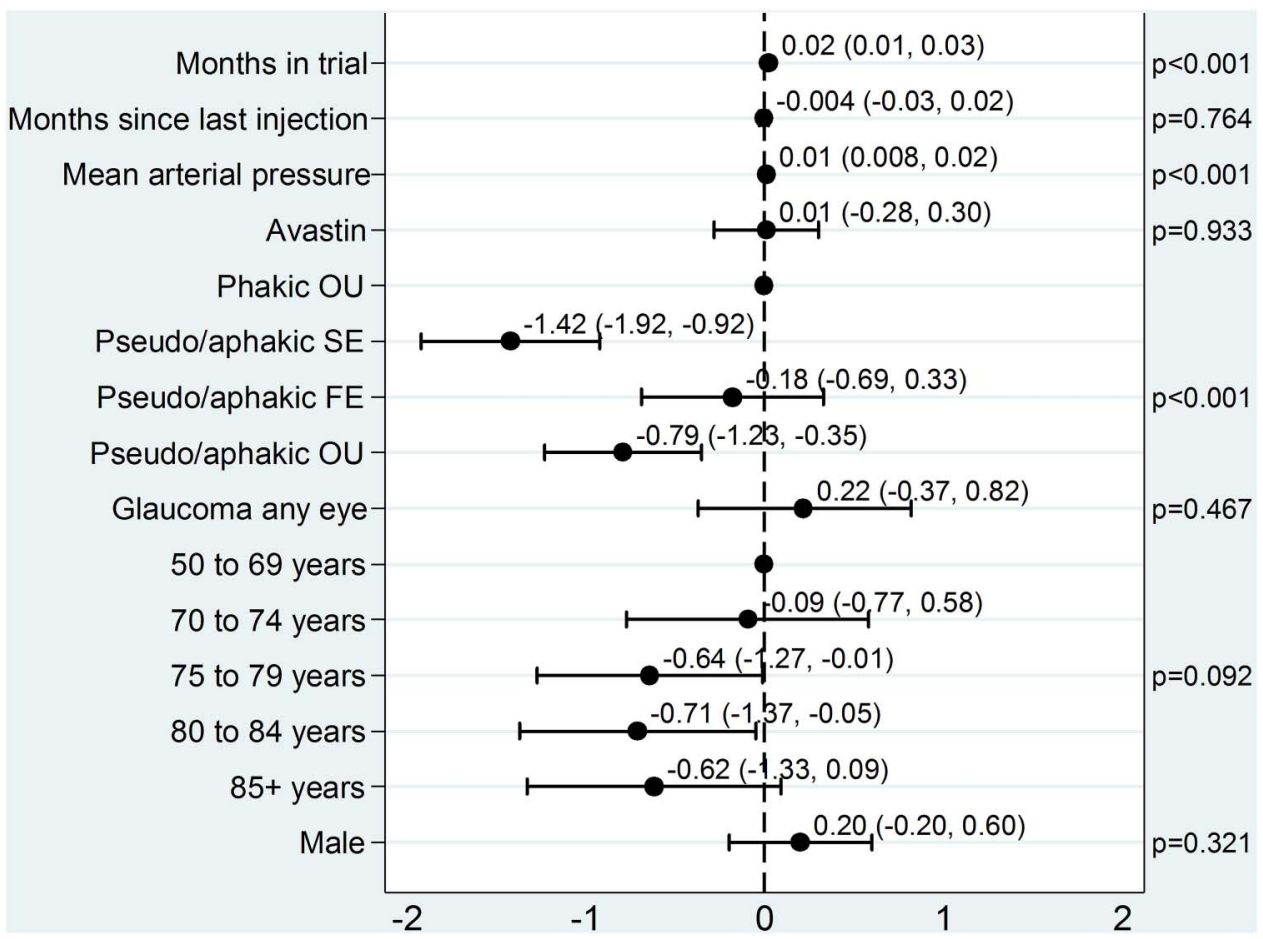

Figure 2 Model for preinjection IOP in the study eye. Plot of the effects of covariates on preinjection IOP in the study eye. The point estimates and $95 \% \mathrm{Cls}$ are shown. FE, fellow eye; IOP, intraocular pressure; OU, both eyes; SE, study eye.

Outcome 3: difference in preinjection IOP between study and fellow eyes during time in study (study eye minus fellow eye)

Preinjection IOP was measured in both eyes for 13208 (98.8\%) of the 13371 attended study visits. Data were available for 606 patients at visit 0 and 510 patients at visit 24 .
Figure 3 shows the multivariable effect estimates of the covariates. The difference between IOP in the two eyes increased over time $(0.01 \mathrm{~mm} \mathrm{Hg} / \mathrm{month})$ with higher readings from the study eye compared with the fellow eye. Longer time intervals between injections reduced the difference in IOP between the study and fellow eye $(-0.02 \mathrm{~mm} \mathrm{Hg})$. Cataract surgery on the

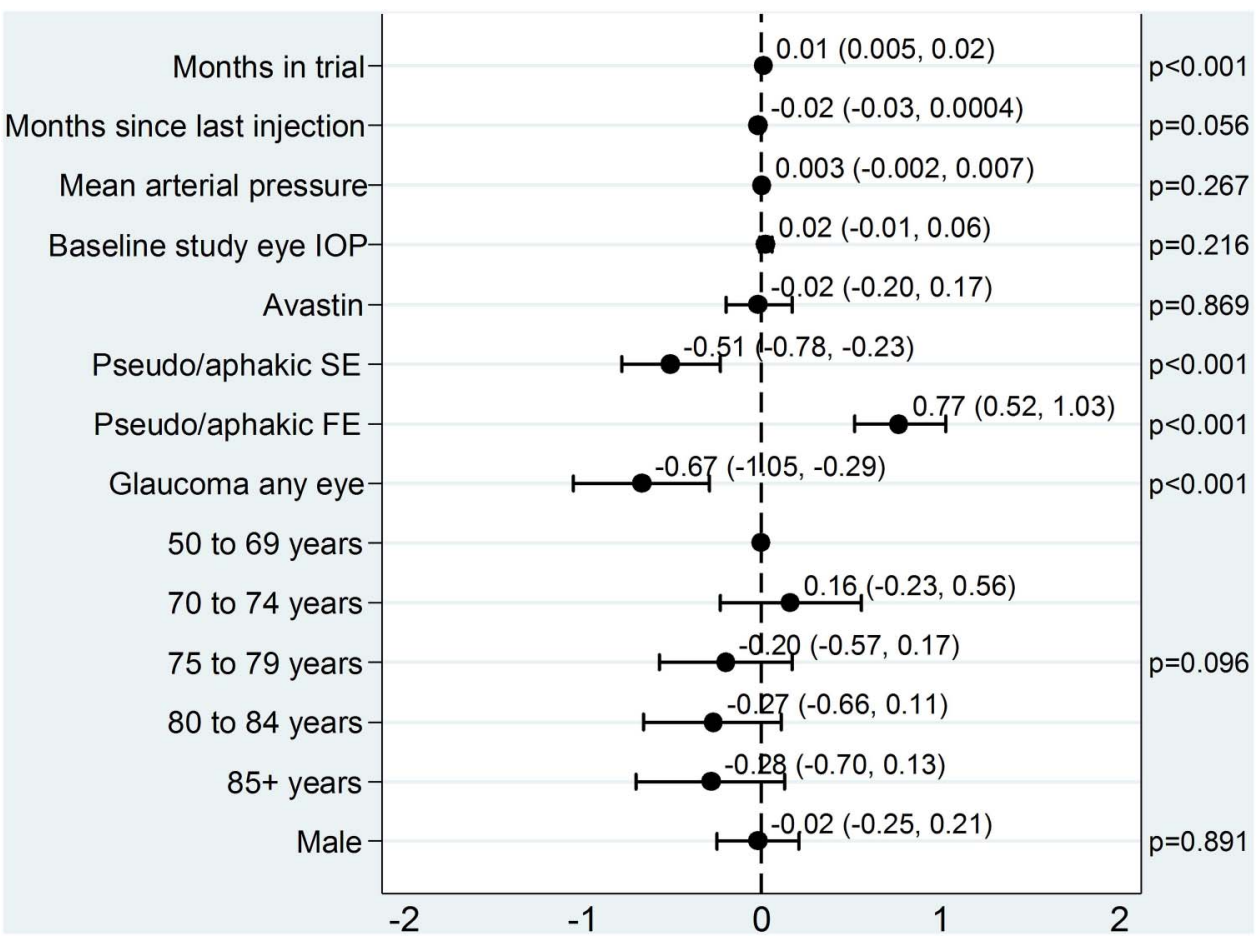

Figure 3 Model for difference in preinjection IOP between the study and fellow eyes (study eye minus fellow eye). Plot of the effects of covariates on difference in IOP between study and fellow eye. The point estimates and $95 \%$ Cls are shown. FE, fellow eye; IOP, intraocular pressure; SE, study eye. 
study eye and glaucoma in any eye significantly reduced the difference in preinjection IOP between eyes $(-0.51$ and $-0.67 \mathrm{~mm} \mathrm{Hg}$, respectively) while that on the fellow eye significantly increased the difference $(0.77 \mathrm{~mm} \mathrm{Hg})$. Mean arterial pressure, baseline IOP in the study eye, study drug, age at randomisation and gender did not have a statistically significant effect on the difference in preinjection IOP between the two eyes $(\mathrm{p}=0.27, \mathrm{p}=0.22, \mathrm{p}=0.87, \mathrm{p}=0.096$ and $\mathrm{p}=0.89$, respectively).

\section{DISCUSSION}

This exploratory analysis on the IVAN trial data has demonstrated a statistically significant increase with time in trial of the preinjection IOP (both study eye only and intereye difference analyses) and the immediate postinjection IOP change in study eyes that received anti-VEGF therapy. It should be noted that the study population was patients with treatment naive nAMD, of whom less than $10 \%$ had glaucoma; investigators were discouraged from recruiting patients with advanced glaucoma. Accordingly, the results of this should not be extrapolated to those with advanced glaucoma.

In our models, we chose time in trial as a proxy for number of injections (we could not fit both as they were highly correlated). In the models dealing with IOP spike and preinjection IOP over time in the study eye, this effect also includes changes with age within patients (which is distinct from the crosssectional effect of age between patients) since patients were ageing with time in trial. The third analysis, of changes in the intereye difference in IOP over time, provides the most direct estimate of the trend in IOP over time attributable to the number of injections, since ageing cannot be a consideration (ie, both eyes age at the same time).

The first model explored was the immediate postinjection IOP change. An injection of even a small volume into the vitreous gives an immediate rise in the IOP, and injections of anti-VEGF agents are no exception ${ }^{9}{ }^{14-16}$ given the non-extensible properties of the sclera. This pressure rise usually returns to normal in under $1 \mathrm{~h}$ without the need for pressure lowering treatment, and therefore our analyses were adjusted for the varying time of the postinjection measurement after administration of the injection.

As adjustment of IOP following a volume load occurs as a consequence of pressure dependent increased flow through the trabecular meshwork (the uveoscleral pathway flux is independent of the ocular pressure), we predicted that damage to the trabecular meshwork would give rise to an elevated and more prolonged postinjection IOP rise. Kahook et $a l^{17}$ raised this as a particular concern for repackaged bevacizumab due to the presence of increased levels of particulates associated with repackaging. Our data found no difference between those receiving ranibizumab and those receiving repackaged bevamizumab arguing against an association between repackaging and pressure rises.

Consistent with previous reports, a small $(1.15 \mathrm{~mm} \mathrm{Hg})$ increase in the pressure rise in study eyes of patients with glaucoma $^{15}$ compared with those without glaucoma was observed. A reduction in IOP of similar size $(1 \mathrm{~mm} \mathrm{Hg})$ is seen in cataract-operated versus unoperated study eyes. Fluid has to flow past the lens from the vitreous cavity to the anterior chamber and then into the angle and out through the trabecular meshwork. Intraocular lenses, due to their smaller size, reduce the resistance to this flow. We would have expected the same effect when both eyes were pseudo/aphakic and it is a matter of surprise that this was not observed.

On average the IOP spike increased at a rate of $0.03 \mathrm{~mm} \mathrm{Hg} /$ month in the trial, which over the 24-month period equates to a rise of $0.72 \mathrm{~mm} \mathrm{Hg}$. There was no suggestion that the IOP increase was higher with bevacizumab.

In the second model, we explored whether a rise in the preinjection IOP would occur in the study eye over time. Three significant factors emerged. The first was phakic status, with our findings being consistent with previous observations that cataract surgery causes a lowering of the ocular pressure. ${ }^{18}$

After allowance for cataract surgery, age and glaucoma, there was a small $(0.02 \mathrm{~mm} \mathrm{Hg} /$ month $)$ significant rise in IOP over time but no relationship with the agent injected.

We repeated the analysis excluding blood pressure from the model as $0.023 \mathrm{~mm} \mathrm{Hg}$ rise in IOP per $\mathrm{mm} \mathrm{Hg}$ rise in blood pressure has been shown ${ }^{19}$ and is 0 similar to the $0.01 \mathrm{~mm} \mathrm{Hg}$ noted in the present study. Anti-VEGF agents can cause a rise in blood pressure, and if the mechanism for their effect on the ocular pressure is mediated by this effect on the blood pressure, then controlling for blood pressure would reduce or eliminate this effect. We checked this and the relationship remained unchanged arguing for a local effect rather than a systemic action.

As IOP tends to rise with age, we constructed a third model using the fellow eye as an internal control, which removes the effect of in-trial ageing. Again, cataract surgery and glaucoma appeared in the model in a manner consistent with the previous analyses. The rise in IOP was similar to that observed in the previous model supporting our findings of a small but significant rise in IOP with injection frequency. As with the other models, there was no difference between the anti-VEGF drugs.

Overall, our data support an ocular hypertensive action of anti-VEGF drugs probably mediated by a direct action on the trabecular meshwork. The endothelial cells are known to express VEGF receptors and endothelial nitric oxide synthase, and one potential mechanism for the observed rise in the IOP is inhibition of the ocular hypotensive VEGF-NO pathway. ${ }^{20}$ There was no indication of differential effects on IOP between ranibizumab and bevacizumab.

The effect on IOP is small but discernible due to the size of this trial. As anti-VEGF therapies require long administration, information on the potential risk of high IOP spikes and chronic IOP elevation becomes increasingly relevant. The other studies that have failed to identify the change in IOP that occurs in eyes treated with anti-VEGF agents have generally had small sample sizes and short-term follow-up. ${ }^{14}$ We cannot comment on the risk of developing glaucomatous optic neuropathy (disc appearances were not graded) but the limited evidence available from the literature so far is reassuring. ${ }^{21-24}$ Nonetheless, a note of caution should be struck as endothelial damage from radiation is a potentially late complication (reflecting the slow turnover rate of endothelial cells) and can take 3 years to manifest ${ }^{25}$ and a similar situation may apply here.

We conclude that repeated use of anti-VEGF agents induces a small but statistically significant rise in IOP in treated eyes.

Contributors All authors have read and approved this manuscript. UC was the lead investigator. FG, JG and AJEF were all trial investigators. BCR, CAR and DMLJS are all members of the trials unit that run the trial. The data analysis for this paper was performed by DMLJS and supervised by CAR and BCR. The manuscript was written by AJEF and approved by all authors.

Funding The IVAN trial was funded by the National Institute for Health Research (NIHR) Health Technology Assessment (HTA) programme (project number 07/36/01). The views and opinions expressed are those of the authors and do not necessarily reflect those of the Health Technology Assessment programme, National Institute for Health Research, the UK National Health Service, or the Department of Health.

Competing interests $C A R$, lecture fees from Novartis; $B C R$, lecture fee from Janssen-Clegg; FG, education grants from Bayer and Novartis and consultancy fees 
from Alimera, Allergan, Bayer and Novartis and research funds from Allergan, Bayer, Novartis, pSivida, Oraya and Sakura; JG, consultancy fees and research funding from Novartis; UC, consultancy fees from Alimera, Bayer, Genentech-Roche, Ophthotech and Oraya Therapeutics. Travel grants from Novartis and Ophthotech. Institution received funding from Allergan, Alcon, Bayer, Genentech-Roche, Notal Vision and Novartis.

Ethics approval 07/NIR03/37.

Provenance and peer review Not commissioned; externally peer reviewed.

Open Access This is an Open Access article distributed in accordance with the Creative Commons Attribution Non Commercial (CC BY-NC 4.0) license, which permits others to distribute, remix, adapt, build upon this work non-commercially, and license their derivative works on different terms, provided the original work is properly cited and the use is non-commercial. See: http://creativecommons.org/ licenses/by-nc/4.0/

\section{REFERENCES}

1 Kim YJ, Sung KR, Lee KS, et al. Long-term effects of multiple intravitreal anti-vascular endothelial growth factor injections on intraocular pressure. Am J Ophthalmol 2014;157:1266-71.

2 Adelman RA, Zheng Q, Mayer HR. Persistent ocular hypertension following intravitreal bevacizumab and ranibizumab injections. J Ocul Pharmacol Ther 2010;26:105-10.

3 Carver J, Bouska C, Corey R. Avastin and risk of glaucoma. New York: Retina Congress, 2009.

4 Wehrli SJ, Tawse K, Levin MH, et al. A lack of delayed intraocular pressure elevation in patients treated with intravitreal injection of bevacizumab and ranibizumab. Retina (Philadelphia, Pa) 2012;32:1295-301.

5 Hoang QV, Tsuang AJ, Gelman R, et al. Clinical predictors of sustained intraocular pressure elevation due to intravitreal anti-vascular endothelial growth factor therapy. Retina 2013;33:179-87.

6 Hoang QV, Mendonca LS, Della Torre KE, et al Effect on intraocular pressure in patients receiving unilateral intravitreal anti-vascular endothelial growth factor injections. Ophthalmology 2012;119:321-6.

7 Mathalone N, Arodi-Golan A, Sar S, et al. Sustained elevation of intraocular pressure after intravitreal injections of bevacizumab in eyes with neovascular age-related macular degeneration. Graefes Arch Clin Exp Ophthalmol 2012;250:1435-40

8 Choi DY, Ortube MC, McCannel CA, et al. Sustained elevated intraocular pressures after intravitreal injection of bevacizumab, ranibizumab, and Pegaptanib. Retina (Philadelphia, Pa) 2011;31:1028-35.

9 Bakri SJ, Pulido JS, McCannel CA, et al. Immediate intraocular pressure changes following intravitreal injections of triamcinolone, Pegaptanib, and Bevacizumab. Eye (Lond) 2009;23:181-5.
10 Kahook MY, Kimura AE, Wong LJ, et al. Sustained elevation in intraocular pressure associated with intravitreal Bevacizumab injections. Ophthalmic Surg Lasers Imaging 2009;40:293-5.

11 Sniegowski M, Mandava N, Kahook MY. Sustained intraocular pressure elevation after intravitreal injection of bevacizumab and ranibizumab associated with trabeculitis. Open Ophthalmol J 2010;4:28-9.

12 Liu L, Ammar DA, Ross LA, et al. Silicone oil microdroplets and protein aggregates in repackaged Bevacizumab and ranibizumab: effects of long-term storage and product mishandling. Invest Ophthalmol Vis Sci 2011;52:1023-34.

13 Chakravarthy U, Harding SP, Rogers CA, et al. Alternative treatments to inhibit VEGF in age-related choroidal neovascularisation: 2-year findings of the IVAN randomised controlled trial. Lancet 2013;382:1258-67.

14 Hollands $\mathrm{H}$, Wong J, Bruen R, et al. Short-term intraocular pressure changes after intravitreal injection of Bevacizumab. Can J Ophthalmol 2007:42:807-11.

$15 \mathrm{Kim} J \mathrm{E}$, Mantravadi AV, Hur EY, et al. Short-term intraocular pressure changes immediately after intravitreal injections of anti-vascular endothelial growth factor agents. Am J Ophthalmol 2008;146:930-4.

16 Mojica G, Hariprasad SM, Jager RD, et al. Short-term intraocular pressure trends following intravitreal injections of ranibizumab (Lucentis) for the treatment of wet age-related macular degeneration. Br J Ophthalmol 2008;92:584.

17 Kahook MY, Liu L, Ruzycki P, et al. High-molecular-weight aggregates in repackaged Bevacizumab. Retina (Philadelphia, Pa) 2010;30:887-92.

18 Poley BJ, Lindstrom RL, Samuelson TW. Long-term effects of phacoemulsification with intraocular lens implantation in normotensive and ocular hypertensive eyes. J Cataract Refract Surg 2008;34:735-42.

19 Dielemans I, Vingerling JR, Algra D, et al. Primary open-angle glaucoma, intraocular pressure, and systemic blood pressure in the general elderly population. The Rotterdam study. Ophthalmology 1995;102:54-60.

20 Ricca AM, Morshedi RG, Wirostko BM. High intraocular pressure following anti-vascular endothelial growth factor therapy: proposed pathophysiology due to altered nitric oxide metabolism. J Ocul Pharmacol Ther 2015;31:2-10.

21 Horsley MB, Mandava N, Maycotte MA, et al. Retinal nerve fiber layer thickness in patients receiving chronic anti-vascular endothelial growth factor therapy. Am J Ophthalmol 2010;150:558-61.

22 Shin HJ, Shin KC, Chung H, et al. Change of retinal nerve fiber layer thickness in various retinal diseases treated with multiple intravitreal antivascular endothelial growth factor. Invest Ophthalmol Vis Sci 2014;55:2403-11.

23 Demirel S, Batioğlu F, Özmert E, et al. The effect of multiple injections of ranibizumab on retinal nerve fiber layer thickness in patients with age-related macular degeneration. Curr Eye Res 2015;40:87-92.

24 Parlak M, Oner FH, Saatci AO. The long-term effect of intravitreal ranibizumab on retinal nerve fiber layer thickness in exudative age-related macular degeneration. Int Ophthalmol 2015;35:473-80.

25 Brown CG, Shields JA, Sanborn G, et al. Radiation retinopathy. Ophthalmology 1982;89:1494-501 